\title{
Eliciting Consumer Preferences using Robust Adaptive Choice Questionnaires
}

\author{
JACOB ABERNETHY, THEODOROS EVGENIOU, OLIVIER TOUBIA, \\ and JEAN-PHILIPPE VERT ${ }^{1}$
}

\footnotetext{
${ }^{1}$ Jacob Abernethy is a graduate student at Toyota Technological Institute at Chicago, Chicago, IL 60637, USA (e-mail: jabernethy@tti-c.org) and was a researcher at INSEAD during this work, Theodoros Evgeniou is an Assistant Professor of Technology Management at INSEAD, Bd de Constance, Fontainebleau 77300, France (Tel: +33 (0)1 607245 46, Fax: +33 (0)1 607455 01, email: theodoros.evgeniou@insead.edu); Olivier Toubia is an Assistant Professor at the Marketing Division at Columbia Business School, 3022 Broadway, Room 522, New York, NY 10027, USA (e-mail: ot2107@columbia.edu); and Jean-Philippe Vert is a Researcher at Ecole des Mines de Paris, 35 rue Saint-Honoré, Fontainebleau 77300, France (e-mail: jean-philippe.vert@mines.org). The authors are listed alphabetically.
} 


\title{
Eliciting Consumer Preferences using Robust Adaptive Choice Questionnaires
}

\begin{abstract}
We propose a framework for designing adaptive choice-based conjoint questionnaires that are robust to response error. It is developed based on a combination of experimental design and statistical learning theory principles. We implement and test a specific case of this framework using Regularization Networks. We also formalize within this framework the polyhedral methods recently proposed in marketing. We use simulations as well as an online market research experiment with 500 participants to compare the proposed method to benchmark methods. Both experiments show that the proposed adaptive questionnaires outperform existing ones in most cases. This work also indicates the potential of using machine learning methods in marketing.
\end{abstract}

Index Terms: Marketing, Data mining, Personalization, Knowledge and data engineering tools and techniques, Interactive systems, Knowledge acquisition, Machine learning, Decision support, Nonparametric statistics.

\section{Introduction}

A central problem in marketing is the understanding of consumers' preferences. Various methods have been developed for this purpose, conjoint analysis $[9,8,18,19,47]$ being 
among the most widely used ones. The approach is based on asking respondents questions about their preferences among products (e.g., "do you prefer product A or product B?") and estimating their preferences for the features (attributes) of the products based on their responses. A key issue in conjoint analysis is the design of the questionnaires. The traditional approach has been to use non-adaptive questionnaires: the questions are determined in advance and are not influenced by the respondents' answers. With the advent of online marketing research, researchers and practitioners have become increasingly interested in adaptive questionnaires [21]: each question for every individual is designed in real time based on his or her responses to earlier questions. Despite their attractiveness, adaptive questionnaires can be subject to endogeneity (as will be illustrated in Section 3.5): questions are influenced by the response errors to earlier answers [22]. As a result, while recently proposed adaptive methods tend to outperform non-adaptive benchmarks when response error is low, they typically do not perform as well when response error is high $[43,44]$ (as is often the case in online environments). This suggests the need for questionnaires that retain the benefits of adaptive interviews while being robust to response error.

In this paper we propose a framework for constructing such questionnaires. It combines some fundamental principles used in experimental design $[10,17,25,32,40]$ and in the recent marketing work of [43, 44], with statistical learning theory [45]. Robustness to response errors is achieved by using complexity control, widely used in machine learning such as in Support Vector Machines and Regularization Networks [41, 45]. We develop 
and test a specific case within the proposed framework using Regularization Networks. We also show how the recently proposed polyhedral estimation method of [44] may be viewed as a special case of our framework, albeit with an arbitrarily small weight on complexity control leading to greater sensitivity to response error. We use simulations as well a field experiment to compare our method to the polyhedral method of [44] and to widely used non-adaptive questionnaire design methods. Both experiments show that the proposed approach performs best in most cases.

The paper is organized as follows. We start in Section 2 with a brief overview of previous related work. We present the proposed framework in Section 3, where we also discuss the relation with previous methods. We then compare the performance of the proposed method to benchmark questionnaires using simulations (Section 4) and an online experiment (Section 5), and conclude in Section 6.

\section{Problem Definition and Related Work}

\subsection{Notations and definitions}

As we use terminology that is standard in marketing but not in machine learning, to facilitate exposition we summarize in Table 1 the definitions of some terms. The underlying model in conjoint analysis is that the utility that a consumer derives from a product (also called a profile) is a function of the attributes of this product (e.g., size, weight, battery life, etc). Each attribute may take different levels which can be continuous (e.g., size in inches) or discrete (e.g., "small" or "large"). We represent a product (profile) by a 
row vector $\mathbf{x}$ which has one entry per continuous attribute and $k$ binary entries for each discrete attribute with $k$ levels (the number of levels may vary across attributes), where all $k$ entries are 0 expect for the one corresponding to the level present in the profile (such representation is called "binary coding" [26]). Our method is developed for continuous attributes, and we use an existing transformation to adapt it to discrete attributes.

The utility derived by a consumer from a product $\mathbf{x}$ may be written as a function $U(\mathbf{x})$. A typical assumption is that the function $U$ is additive $[5,39]$ and linear: $U(\mathbf{x})=\mathbf{x} \cdot \mathbf{w}$. Our approach may be generalized to nonlinear functions using kernels [45]. The vector $\mathbf{w}$ is called the partworths vector.

In order to estimate the partworths vectors w's of a set of consumers, a series of questions is asked to each consumer. Different formats of questions have been developed. We focus on the popular format of "Choice-Based Conjoint Analysis" (CBC) [4, 7, 23, $24,44]$ in which each question asked to each consumer consists of choosing one profile out of a set (i.e., indicating which one has the highest utility). See Figure 2 for an example. For simplicity we first consider binary choices: question $i$ consists of choosing among two profiles $\left\{\mathbf{x}_{i 1}, \mathbf{x}_{i 2}\right\}$. Without loss of generality, we assume that the first profile $\mathbf{x}_{i 1}$ is always selected. We describe the extension to larger choice sets in Section 3 and use four choices per question in our experiments. The set of questions asked to a respondent is called a design, and is represented by a matrix called the design matrix $\mathbf{Z}$ with rows $\mathbf{z}_{i}=\mathbf{x}_{i 1}-\mathbf{x}_{i 2}$. Alternative formats of conjoint questions include rating [47] or ranking [39] sets of profiles, and numerically comparing sets of pairs of profiles [36, 37, 43]. 
The accuracy of the estimated w's is driven by two critical aspects: a) the method used to construct the questionnaires; b) the method used to estimate the respondents' partworths based on their responses to the questionnaires. In this paper, we focus on the first aspect.

\subsection{Previous work on choice-based conjoint (CBC) question- naire design}

\subsubsection{Non-adaptive CBC designs}

The literature on non-adaptive CBC questionnaire design builds primarily on the field of experimental design $[10,17,25,32,40]$. The approach can be summarized as minimizing a norm of the asymptotic covariance matrix of the parameter estimates $\hat{\mathbf{w}}$. If one makes the standard response error assumption that the probability that a consumer with partworth

vector $\mathbf{w}$ will choose profile $\mathbf{x}_{i 1}$ over $\mathbf{x}_{i 2}$ is $P_{i 1}=\frac{e^{\mathbf{x}_{i 1} \cdot \mathbf{w}}}{e^{\mathbf{x}_{i 1} \cdot \mathbf{w}}+e^{\mathbf{x}_{i 2} \cdot \mathbf{w}}}$, then [28] showed that the maximum likelihood estimate of $\mathbf{w}$ is asymptotically normal with mean equal to its true value and covariance matrix equal to the inverse of the information matrix $\Omega$ given by:

$$
\boldsymbol{\Omega}=\left(\sum_{i=1}^{n} \sum_{j=1}^{2} P_{i j} \mathbf{q}_{i j}^{\top} \mathbf{q}_{i j}\right)
$$

where $n$ is the total number of choice questions and $\mathbf{q}_{i j}=\mathbf{x}_{i j}-\sum_{k=1}^{2} \mathbf{x}_{i k} P_{i k} . \quad \Omega$ is also the Hessian of the loss function corresponding to maximum likelihood estimation [30], formulated as (recall that we assume without loss of generality that $\mathbf{x}_{i 1}$ is always 
Table 1: Terms used throughout the paper.

\begin{tabular}{|c|c|}
\hline Term & Definition \\
\hline Attribute & $\begin{array}{c}\text { A product characteristic (or feature) } \\
\text { (e.g., size, price, etc). }\end{array}$ \\
\hline Level & $\begin{array}{c}\text { Each attribute can take } \\
\text { Profile }\end{array}$ \\
\hline various values, each of them called a level.
\end{tabular}


selected):

$$
\hat{\mathbf{w}}=\operatorname{argmin}_{\mathbf{w}} \sum_{i=1}^{n}-\log \left(P_{i 1}\right)
$$

An efficient non-adaptive CBC design is defined as one that minimizes a norm of the asymptotic covariance matrix $\Omega^{-1}$. Different norms lead to different definitions of efficiency. The most widely used norm is the determinant, giving rise to so-called $D$ efficient designs $[4,23,25,26]$. D-efficiency minimizes the volume of the confidence ellipsoid around the maximum likelihood estimate $\hat{\mathbf{w}}[20]$ defined by $\left\{\mathbf{w}:(\mathbf{w}-\hat{\mathbf{w}})^{\top} \mathbf{\Omega}(\mathbf{w}-\right.$ $\hat{\mathbf{w}}) \leq 1\}$

Note that the covariance matrix $\Omega^{-1}$ is a function of the true (unknown) $\mathbf{w}$ through the probabilities $P_{i j}$. Until recently, researchers systematically made the simplifying assumption that $\mathbf{w}=0$ (and hence that all $P_{i j}$ 's are equal), leading to a set of approximate efficiency measures, including $D_{0}$-efficiency which approximates $D$-efficiency. $D_{0}$-efficiency is maximized by orthogonal and balanced designs [1], extensively studied and used in practice. These designs are such that the confidence ellipsoid around the estimated $\mathbf{w}$ - calculated under the assumption that $\mathbf{w}=0$ - is spherical (i.e., the covariance matrix is proportional to the identity matrix). Such designs are available only for specific combinations of the numbers of questions, profiles per question, attributes, and levels per attribute. [7] (see also [23]) provide a method for constructing such designs when they exist. Algorithms have been developed to (approximately) maximize $D_{0}$-efficiency when orthogonal and balanced designs do not exist [12, 25, 26, 29].

Conjoint methods that relax the assumption that $\mathbf{w}=0$ have been recently proposed. 
In particular, $[4,23,24,35]$ consider a non-zero prior on $\mathbf{w}$, obtained in practice from a set of pretest respondents (in which case the designs are adapted between respondents) or from managers' beliefs. This prior may be captured by a point estimate or a probability density function over a set of vectors. Maximizing D-efficiency over the prior on w leads to so-called aggregate customization designs. The main characteristic of aggregate customization designs is utility balance: the profiles in each choice set are close in utility - utility being calculated according to the prior on $\mathbf{w}$ - making the choices "a priori" hard (i.e., the probabilities $P_{i j}$ 's are closer to one another). Intuitively, utility balance increases the information provided by each question. Utility balance has been shown to increase efficiency empirically [4, 23, 24, 35] and theoretically [22].

\subsubsection{Previous work on adaptive CBC designs}

The only published method for adaptively designing CBC questionnaires is the polyhedral method (Poly-Q) of [44]. Note that adaptive conjoint analysis has been made possible only recently, with the development of online marketing research. The main idea behind Poly-Q is that the answer to each choice question may be interpreted as a set of inequality constraints on $\mathbf{w}$ (the constraints reflect the fact that the selected profile has the highest utility in the set). The set of w's that satisfy all the constraints implied by the first $n$ choice questions is a polyhedron defined by:

$$
\mathbf{\Phi}_{n}=\left\{\mathbf{w} \geq 0 ; \mathbf{1} \cdot \mathbf{w}=100 ; \forall i=1 \cdots n, \mathbf{z}_{i} \cdot \mathbf{w} \geq 0\right\}
$$


where $\mathbf{1}$ is a vector of 1 's, $\mathbf{1} \cdot \mathbf{w}=100$ is a scaling constraint, and $\mathbf{z}_{i}=\mathbf{x}_{i 1}-\mathbf{x}_{i 2}$. If $\boldsymbol{\Phi}_{n}$ is non-empty, then $\mathbf{w}$ is estimated by the analytic center [38] of $\boldsymbol{\Phi}_{n}, \hat{\mathbf{w}}_{n} \cdot{ }^{1}$ We describe the general case (in which $\boldsymbol{\Phi}_{n}$ may be empty) in Section 3.5. The question selection method proposed by [44] is such that this polyhedron never becomes empty.

Choosing the $(n+1)^{t h}$ question is equivalent to choosing the next constraint $\mathbf{z}_{n+1}$ that will define the new polyhedron $\boldsymbol{\Phi}_{n+1}$. The polyhedron $\boldsymbol{\Phi}_{n+1}$ may be conceptually compared to the confidence ellipsoid around the estimates used in maximum likelihood estimation (2) (see above). Just like $D_{0}$-efficient designs minimize the confidence ellipsoid around the maximum likelihood estimate and make it spherical, Poly-Q attempts to minimize the volume of $\boldsymbol{\Phi}_{n+1}$ and make it as spherical as possible. Moreover, to achieve this, Poly-Q also uses utility balance just like aggregate customization designs. In particular [44] choose the $(n+1)^{t h}$ question according to the following two criteria:

1. Minimize maximum uncertainty: the new constraint $\mathbf{z}_{n+1}$ should be perpendicular to the longest axis of the current polyhedron $\boldsymbol{\Phi}_{n}$. This will tend to make the next polyhedron $\boldsymbol{\Phi}_{n+1}$ as spherical as possible (see [44] p.119-120 for details). The longest axis of $\boldsymbol{\Phi}_{n}$ can be interpreted as the direction of greatest uncertainty regarding the location of $\mathbf{w}$. It is computed using the eigenvector with the smallest positive eigenvalue of a matrix describing the polyhedron [44].

2. Utility balance: the new constraint $\mathbf{z}_{n+1}$ should go through the analytic center $\left(\hat{\mathbf{w}}_{n}\right)$

\footnotetext{
${ }^{1}$ For a polyhedron written in standard form as $\mathbf{\Phi}=\{\mathbf{w} \geq 0 ; \mathbf{A w}=\mathbf{b}\}$, the analytic center is defined as $\operatorname{argmin}_{\mathbf{w}} \sum_{i=1}^{p}-\ln \left(w_{i}\right)$ subject to $\mathbf{A} \mathbf{w}=\mathbf{b}$ where $p$ is the number of positivity constraints dimensionality of $\mathbf{w}$ in this case.
} 
of the current polyhedron $\boldsymbol{\Phi}_{n}$, that is $\mathbf{z}_{n+1} \cdot \hat{\mathbf{w}}_{n}=0$. [44] (p. 119) argue that this minimizes the expected volume of the polyhedron $\boldsymbol{\Phi}_{n+1}$.

These two criteria, motivated by the theory of non-adaptive experimental design discussed above, will also be used in our framework which we describe next.

\section{Adaptive Design of Robust Choice Questionnaires}

\subsection{Presentation of the method}

Our framework is motivated by the combination of the two experimental design criteria summarized above on the one hand, and statistical learning principles (which we now briefly review) on the other.

A standard approach to conjoint estimation is to maximize some measure of fit. However this may lead to overfitting and the estimates may be sensitive to response errors, especially if they are based on limited data (e.g., few choices per respondent). Statistical learning theory [45], introduced to marketing by [16, 14], has addressed this issue by introducing the fundamental notion that the estimates should reflect a trade off between maximizing fit and minimizing the complexity of the estimated model. In particular, after $n$ questions we estimate $\mathbf{w}$ as the minimizer of a loss function of the following general form:

$$
\min _{\mathbf{w}} L_{n}(\mathbf{w})=\sum_{i=1}^{n} V\left(\mathbf{w},\left\{\mathbf{z}_{i}\right\}\right)+\lambda J(\mathbf{w}),
$$


where $V\left(\mathbf{w},\left\{\mathbf{z}_{i}\right\}\right)$ (with $\mathbf{z}_{i}=\mathbf{x}_{i 1}-\mathbf{x}_{i 2}$ ) measures the fit and $J$ controls (penalizes) the complexity of the partworths. The parameter $\lambda$, called regularization parameter, reflects the trade-off between fit and complexity (we set it to $\frac{1}{n}-$ see below). This parameter may also be chosen using cross-validation or a validation set [45]. Different specifications of $V$ and $J$ lead to different machine learning methods. Our approach can be applied to any loss function (4) that is convex and twice differentiable with respect to $\mathbf{w}$. We will adopt a specific formulation known as Regularization Networks (RN) [41, 45, 15] which leads to closed form solutions that are fast to compute - see below. We note that a special case of (4) widely used in machine learning is Support Vector Machines (SVM) [13, 45]. Our framework does not apply to SVM because that loss function is not twice differentiable, which is a requirement as shown below.

Let us now combine the loss function (4) with the two design criteria outlined at the end of Section 2. Let us assume that $n \geq 1$ questions have been asked thus far, ${ }^{2}$ and that the loss function $L_{n}$ is minimized by our estimate $\hat{\mathbf{w}}_{n}$. Notice that the loss function only depends on the $\mathbf{z}_{i}=\left(\mathbf{x}_{i 1}-\mathbf{x}_{i 2}\right)^{\prime}$ 's. Therefore, for products with real-valued attributes, we only need to determine $\mathbf{z}_{n+1}$ in order to generate the next question (and choose any two profiles such that $\left.\mathbf{z}_{n+1}=\left(\mathbf{x}_{n+1,1}-\mathbf{x}_{n+1,2}\right)\right)$. In creating the next question, we adapt the two criteria outlined at the end of Section 2 as follows:

- Minimize maximum uncertainty: We choose a direction for $\mathbf{z}_{n+1}$ along which the current loss function $L_{n}(\mathbf{w})$ is as flat as possible. The flatness of the loss function

\footnotetext{
${ }^{2}$ Clearly for $n=0$ we have no data hence we cannot use our (or any adaptive) method. As in previous work (e.g., [44]) we design the first question randomly.
} 
is by definition given by its second derivate matrix, the Hessian, and it may be interpreted as a measure of uncertainty in the partworth estimates. For example, in the case of maximum likelihood estimation (see Section 2), the Hessian of the loss function (2) is asymptotically equal to the inverse of the covariance matrix of the estimates [30]. The flattest direction can also be seen as a generalization of the longest axis of the polyhedron in the Poly-Q method of [44].

- Utility balance: We create a question involving a set of products that are "a priori" equally attractive. In the case of binary choices, this implies $\mathbf{x}_{n+1,1} \cdot \hat{\mathbf{w}}_{n}=\mathbf{x}_{n+1,2} \cdot \hat{\mathbf{w}}_{n}$, or $\mathbf{z}_{n+1} \cdot \hat{\mathbf{w}}_{n}=0$.

Let us now formalize these two criteria. The estimated utility vector is the only point $\hat{\mathbf{w}}_{n}$ that satisfies $\nabla L_{n}(\mathbf{w})=0$, where $\nabla$ is the gradient operator. The loss function $L_{n}$ being strictly convex and twice differentiable, its "flatness" (or convexity) around that minimum is given by its second derivative matrix (Hessian) $\left[\nabla^{2} L_{n}\right]_{i, j}:=\frac{\partial^{2} L_{n}}{\partial w_{i} \partial w_{j}}$. More precisely, the convexity along a direction $\mathbf{z}$ is given by $\mathbf{z} \nabla^{2} L_{n} \mathbf{z}^{\top}$.

In order to find the direction of smallest convexity (first criterion) orthogonal to $\hat{\mathbf{w}}_{n}$ (second criterion), we therefore solve the following optimization problem:

$$
\begin{array}{cc}
\min _{\mathbf{z}} & \mathbf{z} \nabla^{2} L_{n}\left(\hat{\mathbf{w}}_{n}\right) \mathbf{z}^{\top} \\
\text { Subject to } & \mathbf{z} \hat{\mathbf{w}}_{n}=0, \quad \mathbf{z z} \mathbf{z}^{\top}=1,
\end{array}
$$

where $\mathbf{z z}^{\top}=1$ is a scaling constraint. After projecting the Hessian matrix onto the hyperplane orthogonal to $\hat{\mathbf{w}}_{n}$ by the equation $B_{n}:=\left(\mathbf{I}_{p}-\frac{\hat{\mathbf{w}}_{n} \hat{\mathbf{w}}_{n}^{\top}}{\hat{\mathbf{w}}_{\mathbf{n}}^{\top} \hat{\mathbf{w}}_{\mathbf{n}}}\right) \nabla^{2} L_{n}\left(\hat{\mathbf{w}}_{n}\right)$, where $p$ 
is the dimensionality of $\mathbf{w}$ and $\mathbf{I}_{p}$ is the $p \times p$ identity matrix, this problem reduces to choosing $\hat{\mathbf{z}}_{n+1}$ as the eigenvector of $B_{n}$ with the smallest positive eigenvalue. Note that both $\hat{\mathbf{z}}_{n+1}$ and $-\hat{\mathbf{z}}_{n+1}$ minimize the loss function, i.e., switching the labels of the profiles $\hat{\mathbf{x}}_{n+1,1}$ and $\hat{\mathbf{x}}_{n+1,2}$ is not consequential.

Thus stated, this strategy is very general and can be applied to any estimation procedure of the form (4) as long as $V$ and $J$ are such that the loss function is convex and twice differentiable. We adopt a specific formulation based on Regularization Networks (RN). RN estimation solves the following minimization problem:

$$
\min _{\mathbf{w}} R_{n}(\mathbf{w})=\sum_{i=1}^{n}\left(1-\left(\mathbf{x}_{i 1}-\mathbf{x}_{i 2}\right) \cdot \mathbf{w}\right)^{2}+\lambda\|\mathbf{w}\|^{2}
$$

where the constant of 1 in the square error plays a scaling role for $\mathbf{w}$. The estimate $\hat{\mathbf{w}}_{n}$ after $n$ questions is [45] $\hat{\mathbf{w}}_{\mathbf{n}}=\left(Z_{n}^{\top} Z_{n}+\lambda \mathbf{I}_{p}\right)^{-1} Z_{n}^{\top} \mathbf{1}_{n}$, where $\mathbf{1}_{n}$ is a vector of 1 's and $Z_{n}$ is the design matrix after $n$ questions (the $i^{\text {th }}$ row of $Z_{n}$ is $\left(\mathbf{x}_{i 1}-\mathbf{x}_{i 2}\right)$ ). The next question is designed using the eigenvector with the smallest positive eigenvalue of:

$$
\left(\mathbf{I}_{p}-\frac{\hat{\mathbf{w}}_{n} \hat{\mathbf{w}}_{n}^{\top}}{\hat{\mathbf{w}}_{\mathbf{n}}^{\top} \hat{\mathbf{w}}_{\mathbf{n}}}\right)\left(Z_{n}^{\top} Z_{n}+\lambda \mathbf{I}_{p}\right) .
$$

Indeed, simple linear algebra shows that $R_{n}$ (Equation (6)) and its derivatives can be written in matrix form as follows: $\nabla R_{n}(\mathbf{w})=2\left(Z_{n}^{\top} Z_{n}+\lambda \mathbf{I}_{p}\right) \mathbf{w}-2 Z_{n}^{\top} \mathbf{1}_{n}$, and $\nabla^{2} R_{n}(\mathbf{w})=2\left(Z_{n}^{\top} Z_{n}+\lambda \mathbf{I}_{p}\right)$. These lead to the expressions above.

In summary, for the RN loss function (6), the proposed conjoint analysis method consists of the following two steps at each iteration (question) n: Step 1: Compute 
the estimate of the partworths $\hat{\mathbf{w}}_{\mathbf{n}}=\left(Z_{n}^{\top} Z_{n}+\lambda \mathbf{I}_{p}\right)^{-1} Z_{n}^{\top} \mathbf{1}_{n} ;$ Step 2: The next question (difference vector $\mathbf{z}_{n+1}$ ) is defined by the eigenvector associated with the smallest positive eigenvalue of the matrix given by Equation (7). Note that all the expressions are in closed form and only require the inversion of a matrix of size equal to the number of partworths (dimensionality of $\mathbf{w}$ ). Hence this method is very fast computationally and can be used, for example, for real time online market research. We label it RN-Q.

\section{$3.2 \quad$ Practical Issues}

\subsubsection{Choosing the parameter $\lambda$ in (4)}

As discussed above (see also [45]), the "trade off" parameter $\lambda$ in (4) is often chosen in practice using a small validation set or using cross-validation. While this is feasible ex post when estimating the partworths, this is not feasible ex ante when designing questions. In this paper we set $\lambda$ to $\frac{1}{n}$, where $n$ is the number of questions. This formulation addresses the concern that $\lambda$ should decrease as the amount of data increases [45]. Future work on how to better (possibly adaptively, using data across respondents) set parameter $\lambda$ may further improve the performance of our method.

\subsubsection{Designing questions with more than 2 profiles}

When more than two profiles per question are needed, we do as in Poly-Q [44]: we consider not only the smallest positive eigenvalue of the Hessian (7) but also the second smallest, third, etc, and the corresponding eigenvectors. For example, to construct a choice set with 
4 profiles as in the experiments, we use 2 difference vectors $\mathbf{z}_{n+1,1}$ and $\mathbf{z}_{n+1,2}$ corresponding to the smallest and second smallest positive eigenvalues of the Hessian matrix (7).

\subsubsection{Designing profiles with discrete attributes}

The approach outlined above generates a continuous difference vector $\mathbf{z}_{n+1}$. In many cases attribute levels are discrete and it is not possible to find two profiles such that $\mathbf{z}_{n+1}=\left(\mathbf{x}_{n+1,1}-\mathbf{x}_{n+1,2}\right)$. As this is not the focus of this paper, we address this issue by simply replicating the approach of [44]. Such replication also makes the empirical comparisons with Poly-Q below fair. Other discrete transformations may be used, but this is beyond the scope of this paper.

The idea behind the method used by [44] is to construct two binary profiles $\mathbf{x}_{n+1,1}$ and $\mathbf{x}_{n+1,2}$ such that $\left(\mathbf{x}_{n+1,1}-\mathbf{x}_{n+1,2}\right)$ is as close as possible to being proportional to $\mathbf{z}_{n+1}$ while preserving utility balance $\left(\left(\mathbf{x}_{n+1,1}-\mathbf{x}_{n+1,2}\right) \cdot \hat{\mathbf{w}}_{n} \sim 0\right)$. This is done in two steps: first two vectors $\mathbf{c}_{n+1,1}$ and $\mathbf{c}_{n+1,2}$ are constructed such that $\mathbf{c}_{n+1,1}-\mathbf{c}_{n+1,2}$ is proportional to $\mathbf{z}_{n+1}$; second two binary profile vectors $\mathbf{x}_{n+1,1}$ and $\mathbf{x}_{n+1,2}$ are created to be as close as possible to $\mathbf{c}_{n+1,1}$ and $\mathbf{c}_{n+1,2}$ while satisfying $\left(\mathbf{x}_{n+1,1}-\mathbf{x}_{n+1,2}\right) \cdot \hat{\mathbf{w}}_{n} \sim 0$.

For the first step [44] select $\mathbf{c}_{n+1,1}$ and $\mathbf{c}_{n+1,2}$ to be maximally different while being within the feasible polyhedron $\boldsymbol{\Phi}_{n}$ of equation (3). Ensuring that the points stay within the polyhedron is important for [44] because it implies that the polyhedron $\boldsymbol{\Phi}_{n}$ never becomes empty. Although this is irrelevant to our framework, in order not to confound the comparisons we follow [44] and use $\mathbf{c}_{n+1,1}=\hat{\mathbf{w}}_{n}+\alpha \mathbf{z}_{n+1}$ and $\mathbf{c}_{n+1,2}=\hat{\mathbf{w}}_{n}-\beta \mathbf{z}_{n+1}$ where $\alpha$ and $\beta$ are chosen as $\alpha=\max \left\{\alpha: \hat{\mathbf{w}}_{n}+\alpha \mathbf{z}_{n+1} \geq 0, \mathbf{z}_{i} \cdot\left(\hat{\mathbf{w}}_{n}+\alpha \mathbf{z}_{n+1}\right) \geq 0, \forall i \leq n\right\}$ (similarly 
for $\beta$ ), where the $\mathbf{z}_{i}$ 's are the profile differences from the previous $n$ questions. We exclude data points which are misclassified by our estimate, that is, for which $\mathbf{z}_{i} \cdot \hat{\mathbf{w}}_{n}<0$.

The second step is achieved by solving two knapsack problems (for $i=1,2$ ): maximize $\mathbf{x}_{n+1, i} \cdot \mathbf{c}_{n+1, i}$ subject to $\mathbf{x}_{n+1, i} \cdot \hat{\mathbf{w}}_{n} \leq M$, where $M$ is a random budget constraint and $\mathbf{x}_{n+1, i}$ is constrained to be a binary vector of the appropriate format. Intuitively, $\mathbf{x}_{n+1,1}$ and $\mathbf{x}_{n+1,2}$ are the binary vectors closest to $\mathbf{c}_{n+1,1}$ and $\mathbf{c}_{n+1,2}$ such that $\mathbf{x}_{n+1,1} \cdot \hat{\mathbf{w}}_{n} \sim$ $M \sim \mathbf{x}_{n+1,2} \cdot \hat{\mathbf{w}}_{n}$. If more than 2 profiles per question are needed, we obtain one pair of points $\mathbf{c}_{n+1, i}$ per eigenvector, and similarly associate one profile $\mathbf{x}_{n+1, i}$ with each point, using the same $\hat{\mathbf{w}}_{n}$ and $M$ in all the Knapsack problems. In our experiments in which the number of profiles per question was 4 , if all the resulting $\mathbf{x}_{n+1, i}$ 's were not distinct, we drew another $M$ and repeated the procedure up to 10 times (for computational efficiency reasons). If the profiles were still not distinct after these 10 draws of $M$, we simply used the non-distinct set of $\mathbf{x}_{n+1, i}$ 's as our question set. This is the procedure used by [44].

\subsection{Relation with Non-Adaptive Experimental Designs}

As reviewed in Section 2, the large literature on experimental designs typically attempts to construct designs that minimize a norm of the asymptotic covariance matrix of the estimates $\left(\boldsymbol{\Omega}^{-1}\right.$ in Equation (1)), which is equal to the inverse of the Hessian of the loss function (2) minimized by maximum likelihood estimation. Let us consider our first design criterion, "minimize maximum uncertainty". It is implemented by selecting the direction of the next question $\mathbf{z}_{n+1}$ as the eigenvector associated with the smallest eigenvalue of 
the Hessian of the loss function (6). We can show that this maximally decreases with each question the largest eigenvalue of the inverse of this matrix. The largest eigenvalue of a matrix being a norm on the set of positive semidefinite matrices, our first criterion may also be interpreted as (greedily) leading towards the minimization of a norm of the inverse of the Hessian of the loss function. Indeed, the Hessian $\nabla^{2} R_{n+1}$ of (6) after the $(n+1)^{t h}$ question is simply $\nabla^{2} R_{n+1}=\nabla^{2} R_{n}+\mathbf{z}_{n+1}^{\top} \mathbf{z}_{n+1}$. If $\mathbf{z}_{n+1}$ is the eigenvector with the smallest positive eigenvalue of $\nabla^{2} R_{n}$ then any eigenvector of $\nabla^{2} R_{n}$ is also an eigenvector of $\nabla^{2} R_{n+1}$ with the same eigenvalue, except $\mathbf{z}_{n+1}$. The latter is also an eigenvector of $\nabla^{2} R_{n+1}$ but with eigenvalue equal to $\Lambda_{n}+\left\|\mathbf{z}_{n+1}\right\|^{2}=\Lambda_{n}+1$ where $\Lambda_{n}$ is the eigenvalue of $\nabla^{2} R_{n}$ corresponding to $\mathbf{z}_{n+1}$. Hence the smallest eigenvalue of $\nabla^{2} R_{n}$ (equal to the inverse of the largest eigenvalue of the inverse of the Hessian) is maximally increased by 1. When combining the first design criterion with the second criterion of utility balance, our method is equivalent to performing a change of (orthogonal) basis with $\hat{\mathbf{w}}_{n}$ as one of the new axes, and applying the first criterion to the projection of the Hessian on the other axes. Note that another interpretation of utility balance is that we restrict the first criterion only to uncertainty regarding the direction of $\mathbf{w}$ (i.e., relative importance of the product attributes), not its norm. Reducing uncertainty along $\hat{\mathbf{w}}_{n}$ would only change the norm of our estimate, not its direction.

Let us now stress two key differences between our method and non-adaptive methods. First, the non-adaptive methods minimize the norm of the asymptotic covariance matrix ex-ante, while we decrease a comparable matrix adaptively based on the additional in- 
formation provided by the respondent's previous choices. Second, and more important, we use complexity control. Because maximum likelihood estimation does not control for complexity, the adaptive approach used in this paper does not appear promising for maximum likelihood estimation. The lack of complexity control would result in designs that are sensitive to response errors, like those obtained from Poly-Q as we show below.

\subsection{Relation with Active Learning}

The problem of designing conjoint questionnaires is comparable to that of active learning

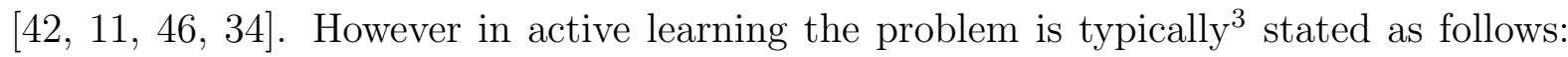
given a (usually small) set of (so called) unlabeled data (e.g., candidate questions to ask), select one for which to ask the label/output (e.g., the dependent variable $y$ in a regression problem). In our case we are not given any set of unlabeled data but instead we design the next data-point/question.

The method we develop here is motivated by principles from the experimental design literature. Interestingly, these principles have been used in active learning as well: for example utility balance has been used in [42], where the data point/question selected at every step is the one for which there is maximal uncertainty regarding the response. There are also active learning methods that attempt to minimize the variance of the estimates, where various measures of variance can be used $[11,34]$. However, we do not know of any active learning method that uses the Hessian of the regularized loss function (4) - which

\footnotetext{
${ }^{3}$ This is a matter of definition. In the most general definition active learning can include our problem as a special case [11] but that is not the typical definition.
} 
includes the complexity control $\|\mathbf{w}\|^{2}$ - while achieving utility balance. Our approach may be adopted to develop a novel active learning method, but we do not develop this avenue further and instead focus on the marketing problem at hand. We also note that an implicit goal of this work is to explore possible links between machine learning and marketing. Future work may explore this interaction further.

\subsection{Relation with Polyhedral Adaptive Choice-Based Conjoint}

\section{Methods}

Our method and that of [44] use the same criteria: (1) minimize the maximum uncertainty on the partworths estimates, (2) utility balance. Formally, in both cases the optimal direction is defined by the smallest positive eigenvector of a matrix describing the space of partworth solutions. However there is a key difference: while we use a regularized loss function (4), the method of [44] does not consider a proper trade off between fit and complexity as we now show.

In its general form, polyhedral estimation consists in finding the analytic center of the polyhedron

$$
\mathbf{\Phi}_{n}=\left\{\mathbf{w} \geq 0, \mathbf{1} \cdot \mathbf{w}=100, \forall i=1 \cdots n, \mathbf{z}_{i} \cdot \mathbf{w} \geq-\delta^{*}\right\}
$$

where $\mathbf{1}$ is a vector of 1 's, $\mathbf{1} \cdot \mathbf{w}=100$ is a scaling constraint, $\mathbf{z}_{i}=\mathbf{x}_{i 1}-\mathbf{x}_{i 2}$ (we again assume binary choices for ease of notation), and $\delta^{*}$ is an estimate of noise obtained by solving

$$
\min _{\mathbf{w}, \delta} \delta
$$


Subject to $\mathbf{z}_{i} \cdot \mathbf{w} \geq-\delta$ for all $i, \quad \mathbf{1} \cdot \mathbf{w}=100, \quad \mathbf{w} \geq 0, \quad \delta \geq 0$

We formalize this 2-stage estimation method within the statistical learning theory framework by writing it as the limit case when $\lambda \rightarrow 0$, of

$$
\begin{array}{rc}
\min _{\mathbf{w}, \delta} & \frac{1}{\lambda} \delta-\sum_{i=1}^{n} \ln \left(\mathbf{z}_{i} \cdot \mathbf{w}+\delta\right)-\sum_{i=1}^{p} \ln \left(w_{i}\right) \\
\text { Subject to } & \mathbf{1}_{p} \cdot \mathbf{w}=100, \quad \delta \geq 0
\end{array}
$$

Hence polyhedral estimation method can be seen as a special case of the general regularized estimation method (4) where fit is measured by $\delta$ and complexity by $-\sum_{i=1}^{n} \ln \left(\mathbf{z}_{i} \cdot \mathbf{w}+\delta\right)-\sum_{i=1}^{p} \ln \left(w_{i}\right)$. (Note that this problem may not be solvable efficiently.) A positive value of $\lambda$ would ensure a proper trade off between fit and complexity. However the estimation method of [44] corresponds to the case when $\lambda \rightarrow 0$ : in this case the optimal solution of (8) is equal to the optimal solution of the 2-stages method of [44] outlined above. Hence the relative weight on fit goes to $+\infty$ in polyhedral estimation. Therefore, a proper trade off between fit and complexity control is not achieved. As a result, the polyhedron defining the feasible estimates, and hence its longest axis and center, are sensitive to the errors in the previous responses. Because this longest axis and center are used to implement the two criteria reviewed above, the adaptive designs of [44] are sensitive to response error. In particular, once a wrong answer is given, the analytic center of the polyhedron will never converge to the true partworths value since the latter will be left outside of all subsequent polyhedra (hence analytic center estimation is in- 
consistent). In contrast, our method reduces the endogeneity issues inherent in adaptive designs ([22]) by introducing a more proper tradeoff between fit and complexity.

We use simulations to illustrate this difference and study the evolution of estimation error as a function of the number of questions. The simulations are designed similarly to the ones we discuss in the next section which are in turn designed based on past studies - see next section. We randomly generated partworths for 500 respondents from a Gaussian with mean $(0.5 \beta, \beta, 1.5 \beta, 2 \beta)$ and diagonal covariance matrix with all diagonal elements equal to $\beta$. Because the method of [44] requires positive partworths, we used the absolute value of the partworths generated from the Gaussian. Using each method (PolyQ and RN-Q), we simulated 16 questions per respondent, each between 4 profiles (with 4 continuous attributes each). We used the logistic model standard in choice modeling $[3,33]$ to simulate the answers: the probability of selecting product $j$ in question $i$ is $P_{i j}=\frac{e^{\mathbf{x}_{i j} \cdot \mathbf{w}}}{\sum_{k=1}^{4} e^{\mathbf{x}_{i k} \cdot \mathbf{w}}}$. With that assumption the parameter $\beta$ in the above Gaussian distribution is a "magnitude" parameter that controls the response error (noise). We chose $\beta$ conservatively such that the probability that an individual makes an error (does not choose the product with the maximum "true" utility) is on average approximately the same as in the high magnitude (low noise) case in the simulations reported in the next section. This leads to $\beta=8$. We measured estimation error using the Root Mean Square Error (RMSE) of the estimated partworths from the true (generated) ones. Both estimated and true partworths were normalized before computing the RMSE such that their absolute values summed to 1 . 


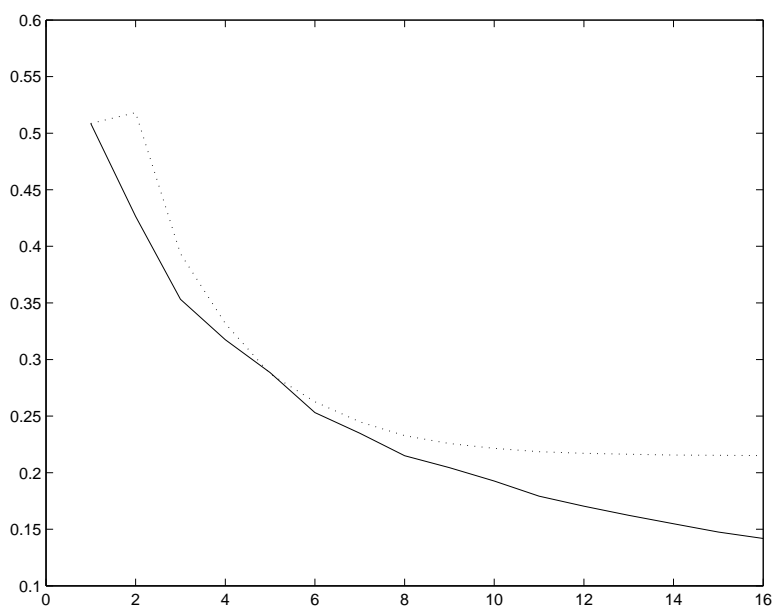

Figure 1: Horizontal axis is the number of questions. Vertical axis is the average RMSE of the estimated utility functions from the true ones (among the 500 individuals). Poly- $Q$ is the dotted line. The RN-based method (RN) is the solid line. The questions are for products with four real-valued attributes (see text).

Figure 1 plots the average RMSE of the estimates as a function of the number of questions used for estimation. We observe that while the accuracy of the RN-Q estimates continually improves after every question, the Poly-Q estimates stop improving after a few questions. As discussed above, once a wrong answer is given to a question, from that point on Poly-Q will not converge to the true partworths vector as it will always try to find an estimate that is in agreement with the wrong answer given. In the theoretical scenario where response error is reduced to exactly 0 (not shown in the figure), this phenomenon is not observed and Poly-Q also converges to the true partworths values. ${ }^{4}$

\footnotetext{
${ }^{4}$ In that case, one could also improve the performance of RN-Q by using a smaller value for $\lambda-$ much like a larger value of $\lambda$ may improve performance if the response error is higher - as known for $\mathrm{RN}$ estimation. However, as noted above, such adjustment of $\lambda$ is not possible a priori when no prior data is available before the questionnaire. The selection of the parameter $\lambda$ is a central and heavily researched problem in machine learning methods like RN or SVM. Future research may further improve the performance of our method through a better selection of $\lambda$.
} 


\section{Simulations}

We now compare empirically the performance of RN-Q to that of previously published adaptive and non-adaptive methods. We use simulations often used in past research (described in this section) as well as an online field experiment (reported in the next section). The design of both experiments follows the marketing tradition.

\subsection{Simulation design}

We compare the performance of the following 4 types of questionnaire design methods (2 adaptive and 2 non-adaptive):

- Orthogonal and balanced designs (i.e., $D_{0}$-efficient designs).

- Aggregate customized designs [4, 23, 35]. Aggregate customization was based on the true mean of the population distribution, and relabeling and swapping were used to improve utility balance. See [4] for details.

- Adaptive designs obtained from Poly-Q.

- Adaptive designs obtained from RN-Q.

For each of these four questionnaires we estimated the partworths using 3 different estimation methods: ${ }^{5}$

- The polyhedral estimation (Poly est) method of [44].

\footnotetext{
${ }^{5}$ The polyhedral estimation method by default uses information about the lowest level for each discrete attribute. We used this information as well for the RN and HB methods, the former by using virtual examples [16] and the latter by re-drawing the partworths from the Metropolis Hastings algorithm until they satisfied the corresponding constraints [2].
} 
- RN estimation (see Equation (6)) (RN est).

- Hierarchical Bayes estimation (HB est) [3, 33, 27], considered the state of the art conjoint estimation method in marketing and widely used in practice. Unlike Poly est and RN est, HB simultaneously estimates the partworths for all respondents hence it is not an individual-level estimation method. Because it uses more information to estimate each individual partworths vector, we expect the HB estimation method to outperform the RN and Poly estimation methods. Recall that the focus of this paper is not on estimation but on questionnaire design. Including HB in our simulations allows us to examine how the proposed questionnaire method performs when coupled with the state of the art estimation method.

Each estimation method may be applied to each type of questionnaire in practice (independent of possible theoretical reasons not to do so). Hence following previous research we consider all the combinations of design $\times$ estimation method. Note that in many commercial applications HB is used as the estimation method irrespective of the questionnaire design method.

We based the design of our simulations on previously published simulation studies. In particular, in order to ensure complete orthogonal and aggregate customization designs we followed $[4,44,16]$ and assumed 16 questions per respondent, each between 4 alternatives described by 4 discrete attributes with 4 levels each, and used logistic probabilities to simulate the answers. Like these other papers, we also considered two levels of response accuracy and two levels of respondent heterogeneity (i.e., how similar respondents' part- 
worths are), giving rise to four conditions. We considered different heterogeneity levels because one of the estimation methods, Hierarchical Bayes, is affected by the level of similarity among respondents.

In each response accuracy $\times$ heterogeneity case, we generated 5 sets of 100 respondents, with partworths drawn from a normal distribution with mean $\left(-\beta,-\frac{1}{3} \beta, \frac{1}{3} \beta, \beta\right)$ for each attribute, and with variance $\sigma_{\beta}^{2}$. The magnitude parameter $\beta$ was set to 0.5 and 2 in the low-accuracy and high-accuracy cells respectively. The parameter $\sigma_{\beta}^{2}$ controls heterogeneity and was set respectively to $\sigma_{\beta}^{2}=0.5 \beta$ and $\sigma_{\beta}^{2}=2 \beta$ in the low and high heterogeneity cells. Those values were used in the published simulations mentioned above and capture the range of response error and heterogeneity observed in practice $[4,44]$. Our performance metric was the Root Mean Square Error (RMSE) of the estimated partworths from the true (generated) ones. Both estimated and true partworths were normalized before computing the RMSE such that the partworths of each attribute summed to 0 and that their absolute values summed to 1.

\subsection{Simulation results}

We summarize the results in Table 2. We compare estimation methods first (for completeness) and question selection methods second (our focus). 
Table 2: Simulation results (RMSE of estimated from true utility functions). Bold numbers indicate best or not significantly different from best at $p<0.05$ for each (magnitude $\times$ heterogeneity $\times$ estimation method) combination. Orth is the orthogonal and balanced design, Cust is the aggregate customized one, Poly-Q is the adaptive design of [44], and $\mathrm{RN}-\mathrm{Q}$ is the proposed one.

\begin{tabular}{|c|c|c|c|c|c|}
\hline Mag & Het & Design & Poly est & RN est & HB est \\
\hline High & Low & Orth & 0.731 & 0.779 & 0.506 \\
& & Cust & 0.641 & 0.601 & 0.394 \\
& & Poly-Q & $\mathbf{0 . 4 7 4}$ & 0.435 & 0.388 \\
& & RN-Q & $\mathbf{0 . 4 7 5}$ & $\mathbf{0 . 4 2 8}$ & $\mathbf{0 . 3 7 6}$ \\
\hline Low & Low & Orth & $\mathbf{0 . 8 0 1}$ & 0.889 & $\mathbf{0 . 7 6 5}$ \\
& & Cust & 0.886 & 0.914 & 0.877 \\
& & Poly-Q & 0.972 & 0.847 & 0.894 \\
& & RN-Q & 0.879 & $\mathbf{0 . 8 1 1}$ & 0.780 \\
\hline High & High & Orth & 0.784 & 0.765 & 0.559 \\
& & Cust & 0.647 & 0.529 & 0.362 \\
& & Poly-Q & $\mathbf{0 . 4 0 6}$ & 0.411 & 0.334 \\
& & RN-Q & 0.413 & $\mathbf{0 . 3 8 3}$ & $\mathbf{0 . 3 2 5}$ \\
\hline Low & High & Orth & $\mathbf{0 . 7 5 7}$ & 0.835 & 0.692 \\
& & Cust & 0.933 & 0.844 & 0.796 \\
& & Poly-Q & 0.831 & 0.739 & 0.746 \\
& & RN-Q & 0.776 & $\mathbf{0 . 7 1 6}$ & $\mathbf{0 . 6 6 4}$ \\
\hline
\end{tabular}




\subsubsection{Estimation}

Our results confirm previous findings (e.g., $[16,44])$ that hierarchical Bayes outperforms other conjoint estimation methods. HB estimates the utilities of all respondents simultaneously, hence implicitly "combining" information across respondents. Unlike HB, the RN and Poly estimation methods are individual-level ones. In our case, HB provides the (significantly) lowest RMSE in all 4 Magnitude $\times$ Heterogeneity cells. In turn, RN estimation performs better than the other individual level estimation method, Poly est: out of the 16 Magnitude $\times$ Heterogeneity $\times$ Question selection method cells, RN estimation is significantly better (at the $p<0.05$ level) in 11, tied in 2 and significantly worse in 3. Note that these significance tests, comparing the first two columns of Table 2 , are not reported in Table 2 .

These results suggest that performance may be improved further by applying our general framework to other loss functions that pool information across respondents. Because HB does not minimize a loss function but rather samples from a posterior distribution, which is not given in closed form, the proposed framework is not directly applicable to HB estimation. Future research may construct estimation methods that combine data from multiple respondents and that are compatible with our framework.

\subsubsection{Question design}

The more relevant comparisons for this paper are the comparisons between questionnaire design methods. The results suggest that the RN-based questionnaire design method is 
the best overall: it is significantly best or tied with best in 8 of 12 Magnitude $\times$ Heterogeneity $\times$ Estimation method cells, and best or tied with best in 3 of the 4 Magnitude $\times$ Heterogeneity cells. (The latter comparisons consider the best performance across all estimation methods.)

We have argued that one of the main contributions of our approach is to produce adaptive choice-based conjoint designs that are robust to response error. Two types of comparisons are possible in order to test this claim. Comparing RN-Q to the other adaptive method (Poly-Q) allows us to evaluate whether $\mathrm{RN}$ based designs are more robust to noise than the other choice-based adaptive designs. Comparing RN-Q to the non-adaptive methods also allows us to evaluate conditions under which the benefits from adaptive questionnaires overweight endogeneity issues.

Let us start with the first comparison. RN-Q is significantly better than Poly-Q in 10 , tied in 1 and significantly worse in 1 of the 12 Magnitude $\times$ Heterogeneity $\times$ Estimation Method cells. More importantly, RN-Q is significantly better than Poly-Q in all 6 low magnitude (high response error) cells. Using HB estimation (the best estimation method), RN-Q performs on average $11.9 \%$ better than Poly-Q in the high-responseerror conditions, while it performs only $2.9 \%$ better on average in the low-response-error conditions.

We finally compare RN-Q to the non-adaptive benchmarks (orthogonal and customized designs). In the high magnitude conditions, RN-Q (as well as Poly-Q - hence both adaptive designs) systematically outperforms both non-adaptive benchmarks, confirming 
the attractiveness of adaptive methods when response error is low, established in previous simulations (e.g., [44]). The more interesting comparisons are when response error is high (low magnitude). RN-based questionnaires perform better than both non-adaptive methods in 3 of the 6 low magnitude $\times$ Heterogeneity $\times$ Estimation method cells and 1 of 2 low magnitude $\times$ Heterogeneity cells. This shows that with RN-based adaptive designs, the benefits from adaptive questionnaires can overweight endogeneity issues even under high levels of response error. RN-based questionnaires achieve the lowest performance relative to non-adaptive benchmarks in the low magnitude $\times$ low heterogeneity condition. In that case, two factors work against adaptive methods as discussed in [44]. First, the endogeneity effects are amplified due to the higher response error. Second, as discussed in [44] and confirmed by our simulations, adaptive methods (Poly-Q and RN-Q) do not perform relatively as well relative to non-adaptive benchmarks (Orth and Cust) under lower levels of heterogeneity, as the similarly among respondents makes it more attractive to use a single questionnaire for everyone.

\section{An Online Experiment}

We next tested the proposed framework using an online field experiment. The sets of question selection methods and partworths estimation methods tested in this experiment were the same as those tested in the previous simulations (question selection methods: Orthogonal design, Aggregate customization, ${ }^{6}$ Poly-Q, and RN-Q; estimation methods:

\footnotetext{
${ }^{6}$ The prior used by aggregate customization was obtained from a pretest involving 50 students from
} 


\section{Choose a Camera}

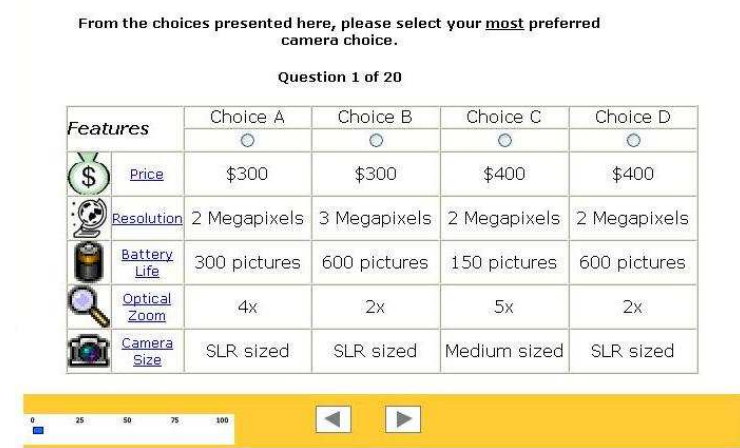

Figure 2: Example Screenshot from the Online Experiment. An example of a choice-based question.

Poly est, RN est, and HB est). 500 respondents from an online panel were randomly assigned to one of the four question selection methods, resulting in 125 respondents per questionnaire. Each respondent completed a 16 question design obtained by the corresponding method, followed by 4 randomly designed holdout questions (the transition from the questionnaire to the holdouts was seamless), a filler task (as a transition), and an external validity ranking task. In this last task the respondents were asked to rank 6 profiles (from most to least preferred), randomly selected from a 16 profile orthogonal and balanced design (different from the one used for the questionnaire). See Figures 2 


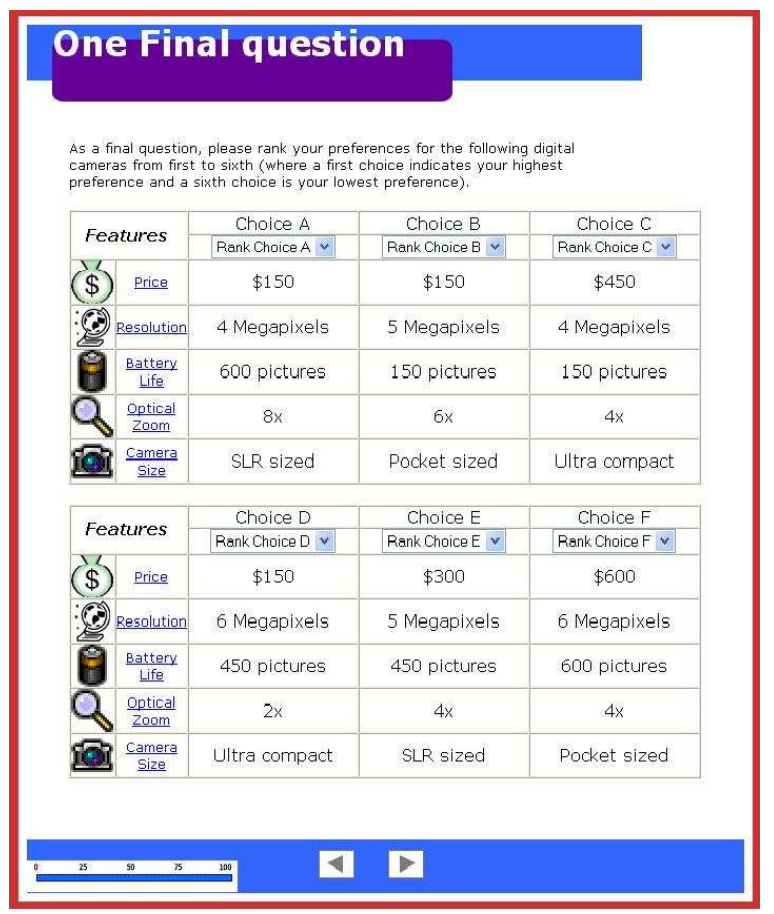

Figure 3: Screenshot from the Online Experiment: The final external validity ranking task.

and 3 for example screenshots from the experiment.

The product chosen for this experiment was digital cameras. We focused on 5 attributes with 4 levels each: Price $(\$ 500, \$ 400, \$ 300, \$ 200)$, Resolution $(2,3,4$, or 5 Megapixels), Battery Life (150, 300, 450, or 600 pictures), Optical Zoom (2x, 3x, 4x, 5x), and Camera Size (SLR, Medium, Pocket, Ultra compact). The attributes were introduced and described to the respondents before the questionnaire. Each choice question comprised 4 profiles.

We measured performance using the following 3 metrics:

1. The percentage of the four holdouts correctly predicted ("Holdout hit rate");

a large US university. 
2. The correlation between predicted and observed rankings in the external validity task ("Choice correlation");

3. The proportion of first choices correctly predicted in the external validity task ("Choice hit rate").

We report the results in Table 3 . In agreement with the simulations and past research, HB was again the best estimation method in all cases, hence for simplicity we only report the performance of the HB estimates for the 4 types of questionnaires. We estimate the partworths based on 3 to 16 questions, and compute the performance of each intermediate estimate. To test the relative performance of the different questionnaires when there are few versus more questions, we report the average performance for questions 3-8 (not including questions 1 and 2, the first being a random one), and questions 9-16. Moreover, we report both the mean and the median (across respondents) of the performance, the latter being less sensitive to possible "outlier respondents".

We use bold numbers in Table 3 to indicate the best performing method in each performance metric $\times(3-8$ vs. $9-16$ questions $) \times($ mean vs. median $)$ combination. Table 3 shows that the proposed question design method is the best in 8 out of the 12 cases, more than any other method. In terms of the median, it is the best in 5 out of 6 cases. A series of pairwise Mann-Whitney U-tests to compare RN-Q to each of the other questionnaires shows that RN-Q is never significantly worse and is significantly better (at the 0.05 level) than each of the other questionnaires in at least one of the (3-8 versus 9-16) $\times$ (performance metric) possible comparisons. In conclusion, the experiment confirms the 
Table 3: Comparison of the question selection methods. Mean and median (across 125 respondents) of the average performance across questions 3-8 and 9-16. The estimation method used is HB. The higher a number the better.

\begin{tabular}{|c||c|c||c|c|}
\hline Holdout & $3-8$, Mean & $3-8$, Median & $9-16$, Mean & $9-16$, Median \\
\hline Orth & 0.47 & 0.46 & 0.60 & 0.63 \\
\hline Cust & 0.49 & 0.50 & 0.60 & 0.63 \\
\hline Poly-Q & $\mathbf{0 . 5 6}$ & $\mathbf{0 . 5 8}$ & 0.60 & 0.63 \\
\hline RN-Q & 0.54 & 0.54 & $\mathbf{0 . 6 1}$ & $\mathbf{0 . 6 6}$ \\
\hline \hline Choice corr & $3-8$, Mean & $3-8$, Median & $9-16$, Mean & $9-16$, Median \\
\hline Orth & 0.48 & 0.61 & $\mathbf{0 . 6 0}$ & 0.73 \\
\hline Cust & 0.45 & 0.52 & $\mathbf{0 . 6 0}$ & 0.73 \\
\hline Poly-Q & 0.45 & 0.53 & 0.52 & 0.64 \\
\hline RN-Q & $\mathbf{0 . 5 0}$ & $\mathbf{0 . 6 5}$ & 0.58 & $\mathbf{0 . 7 6}$ \\
\hline \hline Choice hit & $3-8$, Mean & $3-8$, Median & $9-16$, Mean & $9-16$, Median \\
\hline Orth & 0.40 & 0.33 & 0.54 & 0.63 \\
\hline Cust & $\mathbf{0 . 4 8}$ & $\mathbf{0 . 5 0}$ & $\mathbf{0 . 5 9}$ & 0.63 \\
\hline Poly-Q & 0.43 & 0.33 & 0.49 & 0.50 \\
\hline RN-Q & $\mathbf{0 . 4 8}$ & $\mathbf{0 . 5 0}$ & 0.54 & $\mathbf{0 . 7 5}$ \\
\hline
\end{tabular}

relative advantage offered by the proposed questionnaire design method.

\section{Conclusions}

We have developed a framework for designing robust adaptive choice-based conjoint questionnaires, an important problem in marketing, based on experimental design and statistical learning theory principles. We have shown how to use complexity control to design questionnaires that are less affected by response error and endogeneity. We have validated our framework using simulations as well as a field experiment, and discussed similarities and differences between our method and previous non-adaptive and adaptive ones. The recently proposed adaptive polyhedral choice based conjoint estimation method $[43,44]$ 
can be seen as a special case within our framework, albeit with an arbitrarily small weight on complexity control leading to greater sensitivity to response error.

Various research questions can be explored in the future. For example, one could explore ways of better tuning the parameter $\lambda$ adaptively as respondents answer questions. There are also open theoretical questions, such as the study of the non-asymptotic rate of convergence and the consistency properties of our methods. Another exciting area for future research is the exploration of alternative definitions of complexity that capture the 'cognitive' complexity of making decisions. In particular, a lot of work in consumer research indicates that people use various heuristics for making choices $[31,6]$. Such heuristics may be modeled as penalties or constraints on the estimated utility functions, much along the lines of the standard complexity control penalty $\|w\|^{2}$ used in machine learning. A fundamental premise of large body of work in machine learning supports that constraints (e.g., in the forms of penalties) on the partworths (such as the complexity control $\|w\|^{2}$ ) lead to estimates that are more accurate and robust to noise (see for example [45]). Other constraints (e.g., other complexity controls), based on prior knowledge about how people make choices, may further improve performance.

\section{References}

[1] Addelman, Sidney (1962), "Symmetrical and Asymmetrical Fractional Factorial Plans", Technometrics, 4 (February) 47-58.

[2] Allenby, Greg M., Neeraj Arora, and James L. Ginter (1995) "Incorporating Prior 
Knowledge into the Analysis of Conjoint Studies," Journal of Marketing Research, 32, (May) 152-162.

[3] Allenby, Greg M., and Peter E. Rossi (1999) "Marketing Models of Consumer Heterogeneity", Journal of Econometrics, 89, March/April, p. 57 - 78.

[4] Arora, Neeraj and Joel Huber (2001) "Improving parameter estimates and model prediction by aggregate customization in choice experiments", Journal of Consumer Research, Vol. 28, September 2001.

[5] Ben-Akiva, Moshe and Steven R. Lerman (1985), "Discrete Choice Analysis: Theory and Application to Travel Demand", MIT Press, Cambridge, MA.

[6] Bettman, James R., Mary Frances Luce, and John W. Payne (1998), "Constructive Consumer Choice Processes", Journal of Consumer Research, Vol. 25 (December).

[7] Bunch, David S., Jordan J. Louviere, and Don Anderson (1994), "A Comparison of Experimental Design Strategies for Multinomial Logit Models: The Case of Generic Attributes", working paper, Graduate School of Management, University of California at Davis.

[8] Carroll, Douglas and Paul Green (1995) "Psychometric Methods in Marketing Research: Part I, Conjoint Analysis", Journal of Marketing Research, 32, November 1995, p. 385-391. 
[9] Cattin, Philippe, and Dick R. Wittink (1982), "Commercial Use of Conjoint Analysis: A Survey", Journal of Marketing, 46, p. 44-53.

[10] Chaloner, Kathryn, and Isabella Verdinelli (1995), "Bayesian Experimental Design: A Review", Statistical Science, 10(3), p. 273-304.

[11] Cohn, A. David, Zoubin Ghahramani, and Michael Jordan (1996), "Active Learning with Statistical Models', Journal of Artificial Intelligence Research, 4, pp. 129-145.

[12] Cook, Dennis R., and Christopher J. Nachtsheim (1980), "A Comparison of Algorithms for Constructing Exact E-Optimal Designs", Technometrics, 22(August), p. $315-324$.

[13] Cortes, Corinna and Vladimir Vapnik (1995), 'Support Vector Networks'. Machine Learning 20, 1-25.

[14] Cui, Dapeng and David Curry (2005), "Predicting Consumer Choice Using Support Vector Machines with Benchmark Comparisons to Multinomial Logit", Marketing Science, 24(4), p. 595-615.

[15] Evgeniou, Theodoros, Massimiliano Pontil, and Tomaso Poggio (2000), "Regularization Networks and Support Vector Machines", Advances in Computational Mathematics 13 (2000), p. 1-50.

[16] Evgeniou,Theodoros, Constantinos Boussios, and Giorgos Zacharia (2005) "Generalized Robust Conjoint Estimation", Marketing Science, Vol. 24, No. 3. 
[17] Ford, I., Kitsos, C.P. and Titterington, D.M. (1989) "Recent Advances in Nonlinear Experimental Designs", Technometrics, Vol. 31, p. 49-60.

[18] Green, Paul and V. Srinivasan (1978), "Conjoint Analysis in Consumer Research: Issues and Outlook", Consumer Research, 5, 2, p. 103-123.

[19] Green, Paul and V. Srinivasan (1990), "Conjoint Analysis in Marketing: New Developments with Implications for Research and Practice", Journal of Marketing, 54, 4, p. 3-19.

[20] Greene, William (200), "Econometric Analysis", Prentice Hall.

[21] Hauser, John R., Gerald Tellis, and Abbie Griffin (2006), "Research on Innovation: A Review and Agenda for Marketing Science", (forthcoming) Marketing Science.

[22] Hauser, John R., and Olivier Toubia (2005), "The Impact of Endogeneity and Utility Balance in Conjoint Analysis", Marketing Science, Vol. 24, No. 3.

[23] Huber, Joel, and Klaus Zwerina (1996), "The Importance of Utility Balance in Efficient Choice Designs", Journal of Marketing Research, 33, p. 307-317.

[24] Kanninen, Barbara (2002), "Optimal Design for Multinomial Choice Experiments," Journal of Marketing Research, 36 (May), 214-227.

[25] Kuhfeld, Warren F., Randall D. Tobias, and Mark Garratt (1994), "Efficient Experimental Design with Marketing Applications," Journal of Marketing Research, 31, 4(November) 545-557. 
[26] Kuhfeld, Warren F. (2005), "Marketing Research Methods in SAS", SAS Institute Inc., Cary, NC (USA). Available at http://support.sas.com/techsup/technote/ts722.pdf.

[27] Lenk, Peter J., Wayne S. DeSarbo, Paul E. Green, and Martin R. Young (1996) "Hierarchical Bayes Conjoint Analysis: Recovery of Partworth Heterogeneity from Reduced Experimental Designs," Marketing Science, 15, 173-91.

[28] McFadden, Daniel (1974) "Conditional Logit Analysis of Qualitative Choice Behavior", Frontiers in Econometrics, P. Zarembka, ed. New York: Academic Press, p. $105-142$.

[29] Mitchell, Toby J. (1974) "An Algorithm for the Construction of D-optimal Experimental Designs", Technometrics, 16 (May), p. 203-210.

[30] Newey, Whitney K. and Daniel McFadden (1994), "Large Sample Estimation and Hypothesis Testing", in Handbook of Econometrics, Volume IV, Edited by R.F. Engle and D.L. McFadden, Elsevier Science.

[31] Payne, John W., James R. Bettman, and Eric J. Johnson (1993), "The Adaptive Decision Maker", Cambridge: Cambridge University Press.

[32] Pukelsheim, F. (1993), "Optimal Design of Experiments", Wiley: New York.

[33] Rossi, Peter E., and Greg M. Allenby (2003), "Bayesian Statistics and Marketing", Marketing Science, 22(3), p. 304-328. 
[34] Saar-Tsechansky, M. and Provost, F (2004), "Active Sampling for Class Probability Estimation and Ranking", Machine Learning, 54:2, p. 153-178.

[35] Sandor, Zsolt, and Michel Wedel (2001), "Designing Conjoint Choice Experiments Using Managers' Prior Beliefs", Journal of Marketing Research, 38, 4, p. 430-444.

[36] Sawtooth Software, Inc. (1996), "ACA system: Adaptive conjoint analysis, ACA Manual. Sequim, WA.

[37] Sawtooth Sotware, Inc. (2002), "ACA 5.0 Technical paper", Sequim, WA.

[38] Sonnevend, G. (1985), “An 'Analytic' Center for Polyhedrons and New Classes of Global Algorithms for Linear (Smooth, Convex) Programming", Control and Information Sciences, 84, p. 866-876.

[39] Srinivasan, V. and Allan D. Shocker (1973), "Linear Programming Techniques for Multidimensional Analysis of Preferences", Psychometrica, 38,3, p. 337-369.

[40] Steinberg, D.M., and Hunter, W.G. (1984), "Experimental Design: Review and Comment", Technometrics, 26, p. 71-97.

[41] Tikhonov, A. N., and V. Y. Arsenin (1977), Solutions of Ill-posed Problems, W. H. Winston, Washington, D.C.

[42] Tong, S., and D. Koller (2000), "Support vector machine active learning with applications to text classification", In Proceedings of the Seventeenth International Conference on Machine Learning, Stanford University, CA, USA. 
[43] Toubia, Olivier, Duncan I. Simester, John R. Hauser, and Ely Dahan (2003), "Fast Polyhedral Adaptive Conjoint Estimation", Marketing Science, 22 (3).

[44] Toubia, Olivier, John R. Hauser, and Duncan I. Simester (2004), "Polyhedral methods for adaptive choice-based conjoint analysis", Journal of Marketing Research, Vol. 41, p. $116-131$.

[45] Vapnik, Vladimir (1998), Statistical Learning Theory. New York: Wiley.

[46] Warmuth, M.K., G. Rtsch, M. Mathieson, J. Liao, and C. Lemmen (2002), "Active learning in the drug discovery process", In T.G. Dietterich, S. Becker, and Z. Ghahramani, editors, Advances in Neural information processings systems, volume 14.

[47] Wittink, Dick R. and Philippe Cattin (1989) "Commercial Use of Conjoint Analysis: An Update", Journal of Marketing, 53, 3, p. 91-96. 